\title{
Interaktion zwischen psychosozialer Umwelt und Genetik - wie groß ist die klinische Relevanz?
}

\author{
Interaction Between Psychosocial Environments and Genes - \\ What is the Clinical Relevance?
}

Autoren

Institute
Hans Jörgen Grabe ${ }^{1}$, Christian Schwahn ${ }^{2}$

Klinik für Psychiatrie und Psychotherapie der Ernst-Moritz-Arndt-Universität Greifswald im HANSE-Klinikum Stralsund

2 Zentrum für Zahn-, Mund- und Kieferheilkunde der Universität Greifswald

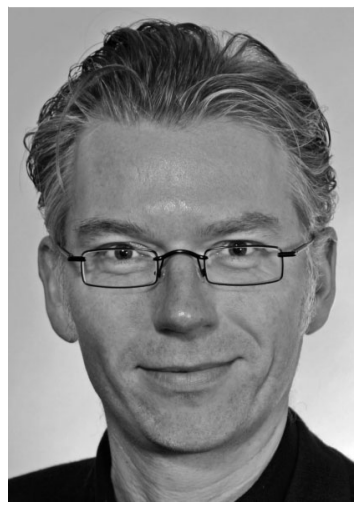

Prof. Dr. med. Hans Jörgen Grabe

\section{Bibliografie}

DOI http://dx.doi.org/ 10.1055/s-0030-1265980

Psychiat Prax 2011; 38: 55-57

(c) Georg Thieme Verlag KG

Stuttgart · New York .

ISSN 0303-4259

\section{Korrespondenzadresse \\ Prof. Dr. med. \\ Hans Jörgen Grabe \\ Klinik für Psychiatrie und \\ Psychotherapie, Ernst-Moritz- \\ Arndt-Universität Greifswald, \\ HANSE-Klinikum Stralsund \\ Rostocker Chaussee 70 \\ 18437 Stralsund \\ grabeh@uni-greifswald.de}

Sehr viele Familien- und Zwillingsstudien deuten auf einen substanziellen biologisch-genetischen Beitrag bei der Entstehung psychischer Erkrankungen hin. Vor allem bipolare Störungen, die Schizophrenie und depressive Erkrankungen stehen im Fokus weltweiter Bemühungen, genetische Risikovarianten zu identifizieren. Gerade der Nachweis von direkten Assoziationen zwischen einer Genvariante und einem klinischen Erkrankungsbild wird häufig von zahlreichen Non-Replikationen begleitet, die Zweifel an der Validität der beschriebenen Assoziationen bedingen. Die genomweiten Assoziationsuntersuchungen (GWAS) haben es in bislang nicht dagewesener Weise ermöglicht, pro Poband oder Patient 500000 bis 1 Mio. Marker über sogenannte GenChips zu analysieren. Aufgrund der hohen A-priori-Wahrscheinlichkeit falsch positiver Befunde, müssen sehr niedrige P-Werte als Schwelle einer statistischen Signifikanz angenommen werden (z.B. 5-mal 10-8). Dadurch ist eine hohe Fallzahl von Personen erforderlich, um bei gegebenen eher kleinen Effektgrößen einzelner genetischer Marker statistisch genomweite Ergebnisse zu erhalten. Gerade bei ätiologisch multifaktoriellen und genetisch heterogenen Erkrankungen sind in der Regel mehrere 10000 Probanden erforderlich, um statistisch abgesicherte Ergebnisse zu erreichen $[1,2]$. Dennoch liefern solche Ergebnisse, wenn diese denn repliziert werden können, wichtige Hinweise auf die biologischen Vulnerabilitätsmechanismen $[3,4]$.

Allerdings ist es mit diesen direkten Assoziationsanalysen nicht möglich, solche Risikomarker zu identifizieren, die erst durch psychobiologischen Stress gewissermaßen „aktiv“ werden. Hierbei geht man davon aus, dass die stressinduzierten biologischen Veränderungen, z.B. eine Aktivierung der Hypothalamus-Hypophysen-Nebennierenrinde-Achse (HPA-Achse) oder der monoaminergen Neurotransmittersysteme, bei vulnerab- len Menschen dazu führen, die Funktionsfähigkeit und Flexibilität dieser Systeme negativ zu beeinflussen. Somit wird die psychobiologische Fähigkeit auf der Verhaltensebene eingeschränkt, die stressinduzierende Situation angemessen zu bewältigen.

Dieses Modell einer Gen-Umwelt-Interaktion entspricht auch oftmals der klinischen Evidenz: Viele Patienten berichten im Vorfeld seelischer Erkrankungen von belastenden Lebensereignissen oder Veränderungen, die sie nur schwer verarbeiten können. Andere Patienten berichten von schwierigen Beziehungserfahrungen in der Kindheit und Jugend, oftmals von traumatisierenden Übergriffen oder von quälender Vernachlässigung durch die Eltern. Diese Erfahrungen führen oft zu einer Beziehungs- und Verhaltensinstabilität, die zum einen auf Entwicklungsdefiziten, aber auch auf erlernten dysfunktionalen Bewältigungsprozessen beruhen. Erst in den letzten Jahren ist vor allem in Tierexperimenten deutlich geworden, wie tiefgreifend und anhaltend neurobiologische Veränderungen bei schon z.T. eher geringen Störungen des „psychosozialen“ Milieus der Tiere sind [5].

Inzwischen belegen zahlreiche Studien eine enge Wechselbeziehung zwischen einer Stressbelastung oder Traumatisierung, biologischer Vulnerabilität und dem Risiko, an einer Stress- oder Traumafolgeerkrankung zu erkranken $[6,7]$.

Wesentliche Pionierarbeit wurde bei der Untersuchung des Short/Long-Promoter-Polymorphismus des Serotonintransportergens (5-HTTLPR) geleistet [8-10]. Trotz unterschiedlicher Expositionsmaße, unterschiedlicher Endpunktdefinitionen und verschiedenster Stichprobencharakteristika konnte in den meisten Studien eine Gen-Umwelt-Interaktion statistisch belegt werden. Bei Studien zur Depression zeigte sich zumeist das Short-Allel als das Risikoallel [11,12]. Davon aus- 
zugehen, dass das Short-Allel des 5-HTTLPR immer als das Risikoallel auftritt, greift allerdings zu kurz. Wahrscheinlich ist es vom Reifungsgrad des Gehirns [13], von der Art der Exposition (Stressbelastung versus Traumatisierung [14]) und vom genetischen Hintergrund abhängig [15], ob das Risiko einer Fehlanpassung des Serotoninsystems mit dem Short- oder mit dem LongAllel assoziiert ist.

Neben diesen Befunden aus dem Serotoninsystem wird eine biologisch plausible, differenzielle Vulnerabilität durch Genvarianten vermittelt, die für relevante Genprodukte innerhalb der endokrinen Stressachse des Körpers (HPA-Achse) codieren. Vor allem kindliche Traumata scheinen zu einer nachhaltigen Veränderung der HPA-Achsen-Regulation beim Menschen aber auch im Tierexperiment zu führen [16]. So konnte unsere Arbeitsgruppe protektive Effekte von Genvarianten des Corticotropin-releasing-Hormon-Rezeptors 1 (CRHR1) nach kindlicher Vernachlässigung bezüglich adulter Depression nachweisen [17]. Auf der anderen Seite vermitteln Genvarianten des FKBP5-Gens eine ausgesprochen hohe Vulnerabilität, an einer PTSD zu erkranken [18]. Wir konnten, unabhängig von komorbider PTSD, ein hohes Risiko für depressive Störungen nach kindlicher Traumatisierung bei dem Vorliegen einer bestimmten FKBP5-Variante nachweisen [19]. Träger der Risikovariante hatten ein 8-fach erhöhtes Erkrankungsrisiko für depressive Störungen (nach DSM-IV).

Gen-Umwelt-Interaktionen werden z.T. über den funktionellen Einfluss von Genvarianten auf neurobiologische Funktionssysteme vermittelt. Zum anderen ist in den letzten Jahren immer deutlicher geworden, dass Umwelteinflüsse über epigenetische Mechanismen wie z.B. die Methylierung von Promotersequenzen, den Funktionszustand von Genen erheblich beeinflussen können [20]. Ohne Zweifel wird die Epigenetik einen wichtigen Beitrag zum Verständnis über die Wechselwirkungen zwischen Umwelt und Genetik leisten können. Weil psychisch relevante Methylierungsprozesse wahrscheinlich primär auf das Gehirn beschränkt sind, ist die Erforschung derartiger Mechanismen beim Menschen sehr schwierig und der Transfer von tierexperimentellen Daten auf den Menschen wird auch hier nicht immer möglich sein.

Die Frage, ob die Erkenntnisse im Bereich der Genetik einen Beitrag zur Früherkennung und ggf. zur differenziellen Therapie psychischer Erkrankungen leisten können, ist oft und kontrovers diskutiert worden. Hier kann die Erforschung von Gen-Umwelt-Interaktionen einen Beitrag leisten: Es ist denkbar, Medikamente zu entwickeln, die bestimmte, in der Folge pathologische Stoffwechselprozesse nach Traumatisierung oder Stressbelastung unterbinden und somit das Risiko einer induzierten Krankheitsdisposition mindern.

Zum anderen stellt sich die Frage, ob die Kenntnis genetischer Risikofaktoren in einer Gruppe exponierter, z.B. traumatisierter, Menschen zu einer besseren Risikoprädiktion beitragen kann. In einer Berechnung basierend auf eigenen Daten zum FKBP5-Gen nach Kindheitstraumatisierung in der Allgemeinbevölkerung zeigen sich durchaus vielversprechende prädiktive Effekte ( $\bullet$ Abb.1). In einem Prävalenzbereich der Depression von 30$40 \%$ beträgt der positive prädiktive Wert des Risikogenotyps über $70 \%$. Hierbei ist natürlich zu berücksichtigen, dass diese Berechnung sich auf die Gruppe der traumatisierten Menschen bezieht. Ohne entsprechende Exposition entfaltet der Risikogenotyp praktisch keine Effekte und ist somit zur Prädiktion untaug-

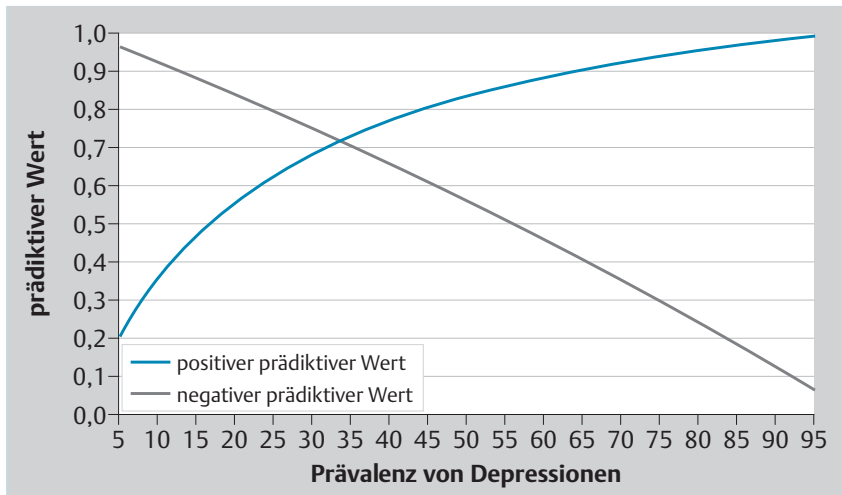

Abb. 1 Positiver und negativer prädiktiver Wert für die Lebenszeitdiagnose einer Depression bei exponierten (Körperlicher Missbrauch in der Kindheit) Risikogenotypträgern des FKBP5-Gens. Diese Berechnung basiert auf einer empirisch ermittelten Sensitivität von 25\% und einer Spezifität von $95 \%$

lich. Also würde ein genetisches Risikoscreening nur in exponierten Populationen Sinn machen. Hierbei wäre allerdings ein Vorscreening auf entsprechende Belastungsfaktoren oder Traumata durchaus praktikabel. Allerdings dürfen methodische Probleme wie ein Responsebias oder die Zeitdauer zwischen Exposition und Krankheitsmanifestation nicht vernachlässigt werden. Mit weiteren genetischen aber auch psychosozialen Risikofaktoren könnten Prädiktionsmodelle weiter verbessert werden. Sinn machen alle Früherkennungsansätze nur, wenn geeignete Interventionsverfahren bereit stehen. Hierbei muss überprüft werden, ob z.B. gezielte psychotherapeutische Interventionen bei exponierten Risikogenotypträgern das Risiko einer späterer Erkrankungen auch tatsächlich senken. Die Forschung hierzu steht erst am Beginn [21].

\section{Literatur}

1 Sullivan PF, de Geus EJ, Willemsen G et al. Genome-wide association for major depressive disorder: a possible role for the presynaptic protein piccolo. Mol Psychiatry 2009; 14: 359-375

2 Lewis CM, Ng MY, Butler AW et al. Genome-wide association study of major recurrent depression in the U.K. population. Am J Psychiatry 2010; 167: 949-957

3 Liu JZ, Tozzi F, Waterworth DM et al. Meta-analysis and imputation refines the association of 15q25 with smoking quantity. Nat Genet 2010; 42: 436-440

4 O'Donovan MC, Craddock NJ, Owen MJ. Genetics of psychosis; insights from views across the genome. Hum Genet 2009; 126: 3-12

5 Rice CJ, Sandman CA, Lenjavi MR et al. A novel mouse model for acute and long-lasting consequences of early life stress. Endocrinology 2008; 149: 4892-4900

6 Gilbert R, Widom CS, Browne Ket al. Burden and consequences of child maltreatment in high-income countries. Lancet 2009; 373: 68-81

7 Heim C, Owens MJ, Plotsky PM et al. Persistent changes in corticotropinreleasing factor systems due to early life stress: relationship to the pathophysiology of major depression and post-traumatic stress disorder. Psychopharmacol Bull 1997; 33: 185-192

8 Caspi A, Sugden K, Moffitt TE et al. Influence of life stress on depression: moderation by a polymorphism in the 5-HTT gene. Science 2003; 301 : 386-389

9 Grabe HJ, Lange M, Wolff B et al. Mental and physical distress is modulated by a polymorphism in the 5-HT transporter gene interacting with social stressors and chronic disease burden. Mol Psychiatry 2005; 10: $220-224$

10 Grabe HJ, Schwahn C, Appel Ket al. Update on the 2005 paper: moderation of mental and physical distress by polymorphisms in the 5-HT transporter gene by interacting with social stressors and chronic disease burden. Mol Psychiatry (in press) 
11 Uher R, McGuffin P. The moderation by the serotonin transporter gene of environmental adversity in the etiology of depression: 2009 update. Mol Psychiatry 2010; 15: 18-22

12 Caspi A, Hariri AR, Holmes A et al. Genetic sensitivity to the environment: the case of the serotonin transporter gene and its implications for studying complex diseases and traits. Am J Psychiatry 2010; 167: 509-527

13 Lau JY, Goldman D, Buzas B et al. Amygdala Function and 5-HTT Gene Variants in Adolescent Anxiety and Major Depressive Disorder. Biol Psychiatry 2009; 65: 349-355

14 Grabe HJ, Spitzer C, Schwahn $C$ et al. Serotonin transporter gene (SLC6A4) promoter polymorphisms and the susceptibility to posttraumatic stress disorder in the general population. Am J Psychiatry 2009; 166: 926-933

15 Pezawas L, Meyer-Lindenberg A, Goldman AL et al. Evidence of biologic epistasis between BDNF and SLC6A4 and implications for depression. Mol Psychiatry 2008; 13: 709-716
16 Heim C, Nemeroff $C B$. The role of childhood trauma in the neurobiology of mood and anxiety disorders: preclinical and clinical studies. Biol Psychiatry 2001; 49: 1023-1039

17 Grabe HJ, Schwahn C, Appel K et al. Childhood maltreatment, the corticotropin-releasing hormone receptor gene and adult depression in the general population. Am J Med Genet B Neuropsychiatr Genet (in press)

18 Binder EB, Bradley RG, Liu Wet al. Association of FKBP5 polymorphisms and childhood abuse with risk of posttraumatic stress disorder symptoms in adults. JAMA 2008; 299: 1291-1305

19 Appel K, Schwahn C, Mahler J et al. Moderation of Adult Depression by a Polymorphism in the FKBP5 Gene and Childhood Physical Abuse in the General Population. Am J Psychiatry (submitted)

20 McGowan PO, Sasaki A, D'Alessio AC et al. Epigenetic regulation of the glucocorticoid receptor in human brain associates with childhood abuse. Nat Neurosci 2009; 12: 342-348

21 Riedel-Heller SG. Sozialpsychiatrie als Forschungsfeld - Antworten auf die Fragen von morgen. Neuropsychiatrie 2009; 23: 249-252 